\title{
Impacto de las alteraciones neuropsicológicas sobre aspectos clínicos en tabaquismo
}

\section{Impact of neuropsychological disorders on clinical aspects of smoking}

\author{
Raquel Martín Ríos*, Ignacio Martín Tamayo**, Francisca lópez-Torrecillas*. \\ * Departamento de Personalidad, Evaluación y Tratamiento Psicológico, Facultad de Psicología. Universidad de Granada. \\ ** Departamento de Metodología de las Ciencias del Comportamiento, Facultad de Psicología. Universidad de Granada.
}

\section{Resumen}

Los estudios que examinan las asociaciones entre las medidas cognitivas y los aspectos clínicos del tabaquismo son limitados y, en general, se limitan a predecir perfiles de riesgo o recaídas. Sin embargo, es esencial comprender la influencia de varias medidas de la función ejecutiva en la adicción a la nicotina a fin indagar factores asociados al mantenimiento del tabaquismo. En el presente estudio se examinó la capacidad de la memoria de trabajo y el descuento por retraso para predecir los años de tabaquismo. La muestra consistió en 180 fumadores que fueron evaluados en la línea de base con medidas de impulsividad cognitiva (Tarea de Descuento de Retraso) y memoria de trabajo [Prueba de Búsqueda y Atención Visual (VSAT) y Secuenciación de Números de Letras (WAIS III)] mientras que la medida de resultado fue los años de adicción. De acuerdo con las predicciones, la memoria de trabajo evaluada con la Prueba de Búsqueda y Atención Visual fue un factor estadísticamente significativo para predecir los años de adicción a la nicotina. Estos hallazgos sugieren que la memoria de trabajo es clínicamente relevante en la dependencia de la nicotina y plantea un patrón de funcionamiento ejecutivo asociado al tabaquismo.

Palabras clave: Fumar; comportamiento adictivo; memoria de trabajo; descuento por retraso.

\begin{abstract}
Studies examining associations between cognitive measures and clinical aspects of smoking are scarce and generally limited to predicting risk profiles or relapses. However, it is essential to understand the influence of several measures of executive function in nicotine addiction in order to investigate factors associated with smoking maintenance. This study examined the ability of working memory and delay discount to predict years of smoking. The sample consisted of 180 smokers who were assessed at baseline with measures of cognitive impulsivity (Delay Discounting Task) and working memory [Visual Search and Attention Test (VSAT) and Letter-Number Sequencing (WAIS III) ] while the outcome measure was years of smoking. Consistent with predictions, working memory evaluated with Visual Search and Attention Test was a statistically significant factor in predicting years of nicotine addiction. These findings suggest that working memory is clinically relevant in nicotine dependence and proposes a pattern of executive functioning associated with smoking.

Keywords: Smoking; addictive behavior; working memory; delay discount.
\end{abstract}




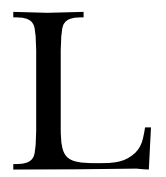

a epidemia del tabaquismo ocupa un lugar preeminente entre las persistentes amenazas para la salud pública a nivel internacional (WHO, 2019). La nicotina se ha revelado como una de las sustancias psicoactivas más adictivas ya que un considerable porcentaje de consumidores se convierten en dependientes (Detandt, Bazan, Quertemont y Verbanck, 2017). Concretamente, los efectos reforzantes de la nicotina inducen la activación del sistema de recompensa cerebral que motiva la probabilidad de repetición del consumo (Carlson, Birkett y Redolar Ripoll, 2018). Por lo que, la adicción a la nicotina representa un trastorno semiológicamente caracterizado por un consumo compulsivo, una paulatina pérdida de control sobre el consumo y la aparición de un cuadro sindromático característico adscrito a la interrupción del uso (Zarrindast y Khakpai, 2019). La consolidación de la dependencia es el resultado de la confluencia entre factores de vulnerabilidad previa y una determinada configuración de mecanismos neurológicos que impulsan cambios neuroadaptativos predominantes en los procesos adictivos (Corominas, Roncero, Bruguera y Casas, 2007). La elevada adictividad y toxicidad derivada del abuso crónico de nicotina modula mecanismos neuronales implicados en funciones cognitivas vitales como la memoria de trabajo, la atención y el control inhibitorio (Zlomuzica et al., 2018). La administración controlada de nicotina ha demostrado atenuar ciertos déficits atencionales, cognitivos y anímicos asociados a la esquizofrenia, déficit de atención/hiperactividad, alzhéimer, párkinson, depresión tardía y daño cognitivo leve (Gandelman et al., 2018; Heishman, Kleykamp y Singleton, 2010; Newhouse et al., 2012). No obstante, el consumo crónico repercute en la funcionalidad de la conectividad y en la coordinación cerebral comprometiendo procesos cognitivos subordinados a dichas estructuras (Durazzo, Meyerhoff y Nixon, 2010). Concretamente, alteraciones en memoria de trabajo, entendida como la capacidad para retener temporalmente información mientras se opera con ella (Wechsler, 1999), podrían favorecer el mantenimiento del consumo al predisponer a pensamientos rumiativos acerca de la droga (Kübler, Murphy y Garavan, 2005). Wagner et al. (2013) examinaron el perfil cognitivo de fumadores y no fumadores con el objetivo de comprobar si los fumadores exhibían déficits cognitivos asociados al consumo crónico. Evaluaron seis dominios: 1) memoria episódica [Prueba auditiva de aprendizaje verbal (AVLT; Helmstaedter, Lendt y Lux, 2001)], 2) atención visual [Trail Making Tests (TMT; Reitan, 1958)] y símbolo de dígitos [Wechsler Adult Intelligence Scale (WAIS; Wechsler, 1999) ], 3) fluidez verbal (tarea de fluidez de letras $\mathrm{S}, \mathrm{A}, \mathrm{B}$ y N), 4) memoria de trabajo evaluada con Digit-span [Wechsler Adult Intelligence Scale (WAIS; Wechsler, 1999)] y secuencia de números (Gold, Carpenter, Randolph, Goldberg y Weinberger, 1997) 5) impulsividad [The Continuous Performance Test (CPT-IP;
Cornblatt, Risch, Faris, Friedman y Erlenmeyer-Kimling, 1988) y 6) interferencia con una tarea «Stroop» (Stroop, 1935). Los resultados reflejaron déficits significativos en atención visual (TMT y Dígitos WAIS) e impulsividad (CPT-IP) en fumadores (Wagner et al., 2013). Más recientemente, Hu et al. (2017) exploraron la relación entre tabaquismo y rendimiento cognitivo en memoria de trabajo entre fumadores y no fumadores evaluados con los subtests de información, aritmética y dígitos [Wechsler Adult Intelligence Scale (WAIS; Wechsler, 1999) ] y en sintomatología ejecutiva [Dysexecutive Questionnaire (DEX; Bodenburg y Dopslaff, 2008)]. Los fumadores reportaron mayores puntuaciones en sintomatología disejecutiva y un menor rendimiento en los subtests de aritmética y dígitos (WAIS) con respecto a los no fumadores. Asimismo, se reflejó una correlación directa entre la memoria de trabajo (Dígitos) y la edad de inicio de fumar (Hu et al., 2018).

Por otro lado, la conducta impulsiva constituye un factor etiológico distinguido en el ámbito de la adicción a la nicotina (Billieux et al., 2010). Una de las dimensiones impulsivas comúnmente exploradas es el descuento demorado (De Wit, 2009) que se evalúa en tareas donde es necesario elegir entre una recompensa inmediata menor o a una recompensa mayor pero demorada (Verdejo-García, Alcázar-Córcoles y Albein-Urios, 2019). Un reciente meta-análisis examinó el rendimiento en diferentes dominios neuropsicológicos entre fumadores y no fumadores concluyendo que los fumadores presentaban mayores tasas en impulsividad cognitiva, lo que vincularon con una preferencia de los fumadores por devaluar recompensas a largo plazo en pos de recompensas inmediatas en tareas de descuento demorado (Conti, McLean, Tolomeo, Steele y Baldacchino, 2019). Además, las tareas de descuento demorado se han impuesto como fuertes predictores de las recaídas tanto en muestras de adultos (González-Roz, Secades-Villa, Pericot-Valverde, Weidberg y Alonso-Pérez, 2019; López-Torrecillas, Perales, Nieto-Ruiz y Verdejo-García, 2014; Reynolds, Richards, Horn y Karraker, 2004) como de adolescentes (Krishnan-Sarin et al., 2007; Sheffer et al., 2014). Sin embargo, hasta donde llega nuestro conocimiento, la investigación acerca de sus aportaciones predictivas en otros aspectos clínicos de tabaquismo es limitada. Asimismo, aunque el tabaquismo ha figurado como objeto de estudio en las últimas décadas, la mayoría de autores se han centrado en la detección de los factores previos que precipitan el inicio de consumo (Gustavson et al., 2017; Harakeh et al., 2012; Lydon, Wilson, Child y Geier, 2014), en las consecuencias adversas del consumo crónico (Billieux et al., 2010; Detandt et al., 2017; Lyvers, Carlopio, Bothma y Edwards, 2014; Sheffer et al., 2014; Valentine y Sofuoglu, 2018) así como en la exploración de variables que comprometen la abstinencia (González-Roz et al., 2019; Harvanko, Strickland, Slone, Shelton y Reynolds, 2019; Krishnan-Sarin et al., 2007; Luijten, Kleinjan y 
Franken, 2016). Por lo que, en comparación con el interés suscitado por otras etapas vinculadas a la dependencia nicotínica, como la abstinencia o el inicio, persiste la escasez de estudios que exploren factores vinculados al uso reiterado del tabaco. Especialmente, investigaciones que reporten acerca de la influencia de variables neuropsicológicas que perpetúen la adicción a la nicotina. Además, se ha constatado que la respuesta terapéutica no es homogénea entre fumadores (Villalbi et al., 2019) en parte porque se desconocen los factores que motivan el uso del tabaco en diferentes colectivos (O’Dell y Torres, 2014). Por lo que, desde el punto de vista clínico y de salud pública, resulta primordial indagar en factores asociados al mantenimiento del tabaquismo que favorezcan el desarrollo de nuevos abordajes terapéuticos. Por esta razón, el objetivo de este estudio pretende aportar evidencia empírica sobre la asociación entre el rendimiento en tareas neuropsicológicas que evalúan memoria de trabajo e impulsividad y los años de adicción en una muestra de fumadores que han solicitado iniciar un tratamiento de deshabituación tabáquica. En base a la evidencia revisada que destaca el potencial rol de la memoria de trabajo en estimular pensamientos rumiantes acerca de la droga, presumimos que las variables que evalúan la memoria de trabajo predecirán en mayor medida los años de adicción.

\section{Método}

\section{Participantes}

La muestra estuvo compuesta por 180 consumidores de tabaco que demandaban tratamiento en el programa de deshabituación tabáquica del Servicio de Prevención de Riesgos Laborales de la Universidad de Granada. Los participantes tenían una media de edad de 47,3 (DT=8,31) dentro de un rango de edad entre 27 y 69 años, de los cuáles $59 \%$ fueron mujeres. La puntuación media de la muestra en el test de Fagerström era de 4,49 (DT=2,32) y el consumo medio de 17,9 (DT=8,94) cigarrillos por día. Los criterios de inclusión fueron: 1) ser consumidor/a de tabaco (Fagerström >3); 2) ser mayor de 18 años; 3) poseer una relación laboral con la Universidad de Granada; 4) participar voluntariamente en el tratamiento ofertado por el Servicio de Prevención de Riesgos Laborales; y 5) cumplimentar debidamente todos los cuestionarios, inventarios y tareas en la evaluación pretratamiento y seguimiento. Los criterios de exclusión fueron: presencia de un trastorno mental grave diagnosticado (trastorno bipolar y/o psicótico, etc.) o que requiera el uso medicamentos de forma regular (ansiolíticos, antidepresivo, etc.), así como dependencia concurrente de otras sustancias (cocaína, heroína, alcohol, etc.). Todos los participantes fueron informados sobre los objetivos del estudio y dieron su consentimiento para participar en el mismo. Previo a la evaluación se infor-

Tabla 1. Características demográficas de la muestra.

\begin{tabular}{|c|c|c|c|c|c|}
\hline Categoría & Medida & Porcentaje & Media & SD & Rango \\
\hline \multirow{2}{*}{ Variables sociodemográficas } & Edad & & 47,3 & 8,31 & $27-69$ \\
\hline & Años de escolarización & & 17,13 & 5,40 & $8-25$ \\
\hline \multirow{4}{*}{ Carrera Profesional } & Personal Administrativo & $62,5 \%$ & & & \\
\hline & Profesorado & $19,5 \%$ & & & \\
\hline & Investigadores & $4 \%$ & & & \\
\hline & Personal de servicio & $14 \%$ & & & \\
\hline \multirow{3}{*}{ Variables tabaco } & Test de Dependencia a la Nicotina Fagerström & & 4,49 & 2,32 & $0-10$ \\
\hline & Severa $($ TFDN >7) & $22 \%$ & & & \\
\hline & Moderada (TFDN<7) & $78 \%$ & & & \\
\hline \multirow{6}{*}{ Marca de cigarrillos } & Rubio & $83,5 \%$ & & & \\
\hline & Negro & $8,5 \%$ & & & \\
\hline & Años de adicción & & 28,43 & 9,84 & 4-57 \\
\hline & Número de cigarrillos al día & & 17,9 & 8,94 & $2-60$ \\
\hline & Nivel de nicotina en mg por cigarillo & & ,99 & ,13 &, $60-1.8$ \\
\hline & Intentos de abandono previo & & 1,27 & 1,35 & $0-12$ \\
\hline \multicolumn{6}{|l|}{ Memoria Trabajo } \\
\hline & Letras y Números (WAIS III) & & 8,70 & 2,90 & $0-15$ \\
\hline & Prueba de búsqueda y atención visual (VSAT) (Estímulos) & & 227,17 & 59,01 & $18-383$ \\
\hline & Prueba de búsqueda y atención visual (Errores) & & 7,34 & 11,31 & $0-95$ \\
\hline Descuento demorado & Tarea ¿Ahora o más Tarde? & &, 57 & ,22 & $0-1$ \\
\hline
\end{tabular}

Nota. $\mathrm{N}=180$ 
mó a los participantes de la voluntariedad del programa, así como del carácter confidencial del tratamiento de los datos (artículo 7 de La Ley 41/2002) realizándose por tanto un consentimiento informado; respaldándonos durante todo el proceso de investigación en el marco legislativo que establece el Código Deontológico (sujeto a la última adaptación de la Ley 25/2009). Además, este estudio cuenta con la aprobación del Comité de Ética en Investigación Humana de la Universidad de Granada.

\section{Instrumentos}

Todas las evaluaciones neuropsicológicas fueron realizadas por psicólogos debidamente formados.

- Letras y Números (Escala de Inteligencia para Adultos de Wechsler, WAIS III; Wechsler, 1999, Adaptación Española TEA Ediciones). En esta prueba se lee al participante una secuencia combinada de letras y números; el participante debe reproducir esta secuencia enunciando primero los números, de menor a mayor, y después las letras, en orden alfabético. Se trata de una tarea en la que intervienen el mantenimiento y la manipulación de información de la memoria de trabajo. La prueba contiene seis elementos donde cada uno se compone de tres secuencias de igual amplitud. Se interrumpe la administración cuando el sujeto falla tres secuencias de un mismo elemento. El número total de aciertos constituye la variable de interés.

- Prueba de búsqueda y atención visual (VSAT; Trenerry, Crosson, Deboe y Leber, 1990). Es un test de búsqueda visual de un objetivo (una letra o símbolo de color) en una matriz destinado a explorar la atención sostenida entendida como la capacidad de activar e inhibir rápidamente respuestas motoras. En este caso, se utilizó la puntuación total de estímulos detectados como variable independiente.

- Tarea de Descuento Demorado. Cuestionario ¿Ahora o más tarde? (Delay Discounting Task, DDT; Kirby, Petry y Bickel, 1999). Se trata de un cuestionario de 27 preguntas de elección monetaria que solicita preferencias entre recompensas más pequeñas e inmediatas o recompensas mayores pero demoradas que varían según su valor y el tiempo de entrega. Se calculó el área bajo la curva (AUC) de acuerdo con la propuesta de Myerson, Green y Warusawitharana (2001). El AUC se calculó para el intervalo de magnitudes de recompensa incluidas en el cuestionario (pequeño-Euro 25-35; medio-Euro 50-60; y grande-Euro 75-85), de acuerdo con la fórmula (x2-x1) [(y1-y2)/2], donde $\mathrm{x} 1 \mathrm{y} x 2$ son sucesivos retrasos, y1 e y2 son los valores subjetivos asociados a estos retrasos (Myerson, Green y Warusawitharana, 2001). La variable predictiva fue el AUC, con valores de AUC más bajos que indican mayor impulsividad.

\section{Patrón de consumo de tabaco}

Entrevista semiestructurada para fumadores (López-Torrecillas, 1996): recopila información sociodemográfica, historia familiar, años de adicción, número de cigarrillos por día e historial de consumo, en la primera sesión del tratamiento. La codificación de la variable criterio «Años de adicción» se definió como el número de años desde la fecha de inicio del consumo hasta el inicio del tratamiento de deshabituación tabáquica.

Test Fagerström de Dependencia a la Nicotina (TFDN) (Fagerstrom y Schneider, 1989)consta de 6 ítems que evalúan el grado de dependencia fisiológica. La puntuación máxima es de 10 puntos y se categoriza en dependencia leve (0-3 puntos), moderada (4-7 puntos) y grave (8-10 puntos).

\section{Procedimiento}

Realizamos un estudio cuasiexperimental, observacional y transversal con un muestreo no aleatorio e incidental, pues solicitaban de manera voluntaria acogerse al programa. Los participantes fueron evaluados de manera individual antes de empezar el tratamiento de deshabituación tabáquica (por lo que las medidas de este estudio corresponden a la evaluación basal), siendo los instrumentos del estudio parte de un protocolo mayor dirigido a la evaluación neuropsicológica dentro de una intervención de deshabituación tabáquica. La intervención de deshabituación tabáquica posterior constaba de tres fases: 1) Fase psicoeducativa (planificación de actividades y establecimiento de objetivos) para reducir el consumo de tabaco y realización de pruebas neuropsicológicas e instrumentos psicológicos; 2) prescripción y administración controlada de vareniclina, fármaco agonista parcial y antagonista en presencia de nicotina de los receptores neuronales para acetilcolina de tipo nicotínico $\alpha 4 \beta 2$; y (3) seguimientos clínicos con estrategias de prevención de recaídas, fomento de hábitos de vida saludables y estrategias destinadas al aumento de la motivación.

El programa comienza con una sesión inicial donde se realiza una entrevista semiestructurada para fumadores (López-Torrecillas, 1996) así como una evaluación neuropsicológica de todos los fumadores, donde se administran las medidas descritas con anterioridad. Esta evaluación inicial se llevó a cabo en una única sesión, teniendo en cuenta los descansos oportunos para evitar el efecto fatiga. Se asignó un código único a los participantes adscritos al programa con la finalidad de realizar un seguimiento individual al tiempo de salvaguardar el anonimato, estableciéndose el responsable del proyecto la persona encargada de custodiar los historiales.

\section{Análisis estadístico}

El análisis estadístico se realizó mediante el programa estadístico IBM SPSS Statistics versión 20.0. En primer 
lugar, se emplearon estadísticos descriptivos para caracterizar a los participantes y correlaciones de Pearson para evaluar las relaciones entre variables con un criterio de significación estadística de $p<, 05$. Para analizar la contribución específica de las variables independientes en los años de adicción se realizó un análisis de regresión jerárquica. Conjuntamente, se realizaron los estadísticos oportunos para la comprobación de supuestos que exige la aplicación del modelo de regresión.

\section{Resultados}

Para comprobar si existe asociación entre las variables se realizó la Prueba de Pearson que nos muestra que en nuestro estudio las variables que evalúan la memoria de trabajo (WAIS y VSAT) presentan una correlación inversa con la variable criterio, siendo una relación leve para la prueba WAIS $(\mathrm{r}=-, 020 ; \mathrm{p}=, 05)$ y moderada para VSAT $(\mathrm{r}=-, 415 ; \mathrm{p}=, 05)$. En este sentido, los fumadores con puntuaciones bajas en las pruebas de memoria de trabajo tienden a presentar más años de adicción. Por otra parte, en relación a la variable que evalúa el descuento demorado (Delay Discounting Task, DDT) se observa una relación directa leve con la variable criterio $(\mathrm{r}=-, 084 ; \mathrm{p}=, 05)$. Por lo que, en la medida que los fumadores descuentan más en esta prueba, es decir, muestran una conducta más impulsiva, los años de adicción aumentan (Tabla 2).

Tabla 2. Matriz de correlaciones entre variable criterio $e$ independientes.

\begin{tabular}{lcccc}
\hline Variables & Años & WAIS & VSAT & AUC \\
\hline Años & 1 & & & \\
WAIS &,- 020 & 1 & & \\
VSAT &,$- 415^{\star}$ &, $162^{\star}$ & 1 & \\
TDD &, 084 &,- 022 &,- 043 & 1 \\
\hline
\end{tabular}

Nota. $\mathrm{N}=180$. Años $=$ Número de años de adicción; WAIS: Puntuación total en subtest Letras y Números de la Escala de Inteligencia para Adultos Wechsler, WAIS III; VSAT= Total de estímulos en la Prueba de búsqueda y atención visual; TDD $=$ Puntuación total en la Prueba de Ahora o Más Tarde. * $\mathrm{P}<.05$.

En lo que respecta al análisis de regresión jerárquica (Tabla 3) se observó que las variables que evaluaban la memoria de trabajo (WAIS y VSAT) explicaron un 17,5\% de la varianza. Finalmente, en el tercer paso, al incorporar la variable AUC que evaluaba el descuento por demora, el modelo explicó un $17,9 \%$ de la varianza criterio, dejando un modelo final estadísticamente significativo, $\mathrm{F}_{(3,176)}$ $=12,80 ; \mathrm{p}<, 000$. En el modelo final, únicamente la variable de memoria de trabajo VSAT resultó estadísticamente significativa $(\beta=-, 070, \mathrm{p}<, 000)$. En este sentido, en la ecuación de regresión se puede observar que la variable puntuación en VSAT representa la variable independiente con mayor peso en la ecuación de regresión. Un incremento de una unidad de desviación en VSAT determina un aumento de
Tabla 3. Análisis de Regresión Jerárquica.

\begin{tabular}{llllllll}
\hline Variable & $\mathbf{R}$ & $\mathbf{R}^{\mathbf{2}}$ & $\mathbf{g l}$ & $\boldsymbol{\beta}$ & $\mathbf{t}$ & $\mathbf{p}$ & $\mathbf{9 5 \%} \mathbf{I C} \mathbf{d e} \boldsymbol{\beta}$ \\
\hline Paso 1 & & & & & & & \\
\hline WAIS &, 020 &, 000 & 1 &,- 069 &,- 273 &, 786 &,$- 570-, 432$ \\
\hline Paso 2 & & & & & & & \\
\hline WAIS & & & &, 163 &, 697 &, 487 &,$- 299-, 626$ \\
VSAT & .418 &, 175 & 1 &,- 071 & $-6,113$ &, 000 &,$- 093--, 048$ \\
\hline Paso 3 & & & & & & & \\
\hline WAIS & & & &, 167 &, 049 &, 478 &,$- 296-, 630$ \\
VSAT & & & &,- 070 &,- 420 &, 000 &,$- 093--, 047$ \\
TDD &, 423 &, 179 & 1 & 2,871 &, 067 &, 329 & $-2,919-8,66$ \\
\hline
\end{tabular}

Nota. $\mathrm{N}=180$. WAIS: Puntuación total en subtest Letras y Números de la Escala de Inteligencia para Adultos Wechsler, WAIS III; VSAT= Total de estímulos en la Prueba de búsqueda y atención visual; TDD= Puntuación total en la Prueba de Ahora o Más Tarde.

* $P<.05$.

los años de adicción de ,420. Por último, al observar la amplitud de los intervalos de confianza de los coeficientes de regresión parcial nos encontramos con estimaciones más ajustadas en las pruebas de memoria de trabajo (VSAT y WAIS), pero mayor amplitud de intervalo en la prueba de impulsividad (Delay Discounting Task, DDT) lo que apuntan a menor precisión a la hora de estimar el valor poblacional de este coeficiente de regresión.

\section{Discusión}

El objetivo de este estudio perseguía explorar la relación entre los años de adicción a la nicotina y el desempeño en tareas neuropsicológicas en fumadores que solicitaban iniciar un tratamiento de deshabituación tabáquica. Los resultados indican que tareas de memoria de trabajo (WAIS y VSAT) junto con una tarea de descuento demorado conforman un modelo de regresión significativo que explica un $17,9 \%$ de la varianza criterio. No obstante, únicamente la variable VSAT representa el principal predictor de los años de adicción en nuestra muestra. Exhibiendo una correlación inversa que indica que a menor rendimiento en VSAT más años de adicción. Hallazgos afines a los reportados por Wagner et al. (2013) que evidenciaron déficits significativos en atención visual en fumadores con respecto a no fumadores (Wagner et al., 2013). Además, concuerdan con la hipótesis funcional-estructural que declara una superposición de los efectos estructurales crónicos del tabaquismo a los efectos funcionales de la administración aguda de nicotina (Sutherland et al., 2016). En consecuencia, las investigaciones que atribuyen propiedades beneficiosos a la nicotina recurren a colectivos distinguidos por un sistema colinérgico alterado o una disfunción subyacente de nAChR (Gandelman, Newhouse y Taylor, 2018). Específicamente, la administración de nicotina controlada promueve mejoras de actividad funcional en la corteza prefrontal (CPF) lateral, corteza cingulada anterior (ACC), tálamo y 
cuneus, es decir, regiones tradicionalmente asociadas a la atención, memoria de trabajo y la ejecución de tareas que demandan procesamiento orientado al exterior (Red de control ejecutivo) (Gandelman et al., 2018; Sutherland et al., 2015). No obstante, el consumo crónico de nicotina entraña alteraciones en el rendimiento cognitivo (Durazzo et al., 2010)así como neuroadaptaciones que culminan en disminuciones en la materia gris de regiones comúnmente identificadas en procesos asociados con la adicción tales como: CPF ventromedial, ínsula, tálamo y cerebelo (Sutherland et al., 2016). Específicamente, la ínsula como sustrato adscrito en el control cognitivo y atencional (Bi et al., 2017) e involucrado en la necesidad de fumar (Paulus y Stewart, 2014) parece desempeñar un papel crítico en el mantenimiento del tabaquismo (Sutherland y Stein, 2018). Además, las diferencias estructurales en la ínsula entre fumadores y controles se advierten de manera más plausible en fumadores con historiales de exposición al tabaquismo más amplios (Li et al., 2015). Como nuestros resultados pretenden aportar datos conductuales a la disquisición sobre las alteraciones estructurales resultantes del impacto nocivo del tabaquismo, como hipótesis inferencial proponemos que los fumadores con mayor historial de adicción muestran rendimientos deficitarios en tareas demandantes de memoria de trabajo y de control atencional que predisponen a pensamientos de deseo rumiante tradicionalmente vinculados a la adicción (Hester y Garavan, 2009; Kübler et al., 2005).

Por otra parte, la tarea de descuento demorado (Delay Discounting Task, DDT) no presentó significación estadística lo que puede revelar que dicha variable intervenga notoriamente en otras etapas de la adicción. Presunción acorde con la hipótesis que sostiene Reynolds y Fields (2012) que afirma que las diferentes dimensiones de impulsividad pueden desempeñar diversas influencias en el inicio del consumo o en etapas posteriores (Reynolds y Fields, 2012). En este caso, el descuento demorado ha sido ampliamente vinculado con la respuesta al tratamiento (González-Roz et al., 2019; Krishnan-Sarin et al., 2007; López-Torrecillas et al., 2014; Reynolds et al., 2004; Sheffer et al., 2014). En resumen, la investigación refiere que, pese a que coexisten diferencias individuales que facilitan la iniciación o el riesgo de recaídas, los efectos de la nicotina en aspectos cognoscitivos sugieren que existe una heterogeneidad en las causas que mantienen el tabaquismo (Bedi et al., 2011; García-Rivas y Deroche-Gamonet, 2019; Hall et al., 2015).

Nuestros resultados ponen de manifiesto que procesos cognitivos demandantes de memoria de trabajo, como la atención sostenida y la búsqueda visual, parecen incidir en el mantenimiento del tabaquismo. Sin embargo, se requiere señalar algunas limitaciones tales como la naturaleza de la muestra ya que, al tratarse de una recolección no aleatoria e incidental, limita la generalización de los resultados. Además, la naturaleza transversal del estudio obstaculiza la obtención de datos que reporten el desarrollo temporal y la relación causal inferida entre las variables. Por lo que se resalta la importancia de implementar diseños longitudinales que permitan dar cuenta de la evolución de la dependencia. Finalmente, el interés que pueda suscitar este estudio trata de clarificar las implicaciones de variables neuropsicológicas en aspectos clínicos de la conducta de fumar tan relevantes y herméticos como el mantenimiento del hábito. Identificar variables mediadoras que motivan el uso de la nicotina resulta crucial a fin de desarrollar estrategias terapéuticas más eficaces (O’Dell y Torres, 2014) que inviten a la inclusión de variables neuropsicológicas. En consecuencia, nuestros resultados deben interpretarse como punto de partida hacia nuevas investigaciones que indaguen la implicación de variables cognoscitivas tanto en el mantenimiento del tabaquismo crónico como sus posibles complicidades en el camino hacia la abstinencia. Puesto que la capacidad predictiva de factores neurocognitivos se ha explorado puntualmente en el abandono del hábito de fumar (Luijten et al., 2016) pese a representar un objetivo prioritario en las políticas preventivas (Villalbi et al., 2019).

\section{Conflicto de intereses}

Los autores declaran no tener ningún conflicto de intereses.

\section{Agradecimientos}

Los autores agradecen a los participantes de este estudio su implicación y participación.

\section{Referencias}

Bedi, G., Preston, K. L., Epstein, D. H., Heishman, S. J., Marrone, G. F., Shaham, Y. y De Wit, H. (2011). Incubation of cue-induced cigarette craving during abstinence in human smokers. Biological Psychiatry, 69, 708-711. doi:10.1016/j.biopsych.2010.07.014.

Bi, Y., Yuan, K., Guan, Y., Cheng, J., Zhang, Y., Li, Y.,... Tian, J. (2017). Altered resting state functional connectivity of anterior insula in young smokers. Brain Imaging and Behavior, 11, 155-165. doi:10.1007/s11682-016-9511-z.

Billieux, J., Gay, P., Rochat, L., Khazaal, Y., Zullino, D. y Van der Linden, M. (2010). Lack of inhibitory control predicts cigarette smoking dependence: Evidence from a non-deprived sample of light to moderate smokers. Drug and Alcohol Dependence, 112, 164-167. doi:10.1016/j. drugalcdep.2010.06.006.

Bodenburg, S. y Dopslaff, N. (2008). The dysexecutive questionnaire advanced. The Journal of Nervous and Mental Disease, 196, 75-78. doi:10.1097/NMD.0b013e31815faa2b. 
Carlson, N. R., Birkett, M. A. y Redolar Ripoll, D. (2018). Fisiología de la conducta. En N. R. Carlson y M. A. Birkett (Eds.), Fisiología de la conducta $\left(17^{a}\right.$ ed.). Madrid: Pearson Educación.

Conti, A. A., McLean, L., Tolomeo, S., Steele, J. D. y Baldacchino, A. (2019). Chronic tobacco smoking and neuropsychological impairments: A systematic review and meta-analysis. Neuroscience and Biobehavioral Reviews, 96, 143-154. doi:10.1016/j.neubiorev.2018.11.017.

Cornblatt, B. A., Risch, N. J., Faris, G., Friedman, D. y Erlenmeyer-Kimling, L. (1988). The continuous performance test, identical pairs version (CPT-IP): I. new findings about sustained attention in normal families. Psychiatry Research, 26, 223-238. doi:10.1016/01651781(88)90076-5.

Corominas Roso, M., Roncero Alonso, C., Bruguera Cortada, E. y Casas Brugué, M. (2007). Sistema dopaminérgico y adicciones. Revista de Neurología, 44, 23. doi:10.33588/ rn.4401.2006222.

De Wit, H. (2009). Impulsivity as a determinant and consequence of drug use: A review of underlying processes. Addiction Biology, 14, 22-31. doi:10.1111/j.13691600.2008.00129.x.

Detandt, S., Bazan, A., Quertemont, E. y Verbanck, P. (2017). Smoking addiction: The shift from head to hands: Approach bias towards smoking-related cues in low-dependent versus dependent smokers. Journal of Psychopharmacology, 31, 819-829. doi:10.1177/0269881117699606.

Durazzo, T. C., Meyerhoff, D. J. y Nixon, S. J. (2010). Chronic cigarette smoking: Implications for neurocognition and brain neurobiology. International Journal of Environmental Research and Public Health, 7, 3760-3791. doi:10.3390/ijerph7103760.

Fagerstrom, K. O. y Schneider, N. G. (1989). Measuring nicotine dependence: A review of the Fagerstrom Tolerance Questionnaire. Journal of Behavioral Medicine, 12, 159-182. doi:10.1007/BF00846549.

Gandelman, J. A., Kang, H., Antal, A., Albert, K., Boyd, B. D., Conley, A. C.,... Taylor, W. D. (2018). Transdermal nicotine for the treatment of mood and cognitive symptoms in nonsmokers with late-life depression. Journal of Clinical Psychiatry, 79. doi:10.4088/JCP.18m12137.

Gandelman, J. A., Newhouse, P. y Taylor, W. D. (2018). Nicotine and networks: Potential for enhancement of mood and cognition in late-life depression. Neuroscience Ẽ Biobehavioral Reviews, 84, 289-298. doi:10.1016/j.neubiorev.2017.08.018.

Garcia-Rivas, V. y Deroche-Gamonet, V. (2019). Not all smokers appear to seek nicotine for the same reasons: Implications for preclinical research in nicotine dependence. Addiction Biology, 24, 317-334. doi:10.1111/ adb. 12607.

Gold, J. M., Carpenter, C., Randolph, C., Goldberg, T. E. y Weinberger, D. R. (1997). Auditory working memory and Wisconsin card sorting test performance in schizophrenia. Archives of General Psychiatry, 54, 159-165. doi:10.1001/archpsyc.1997.01830140071013.

González-Roz, A., Secades-Villa, R., Pericot-Valverde, I., Weidberg, S. y Alonso-Pérez, F. (2019). Effects of delay discounting and other predictors on smoking relapse. Spanish Journal of Psychology, 22, e9. doi:10.1017/ sjp.2019.11.

Gustavson, D. E., Stallings, M. C., Corley, R. P., Miyake, A., Hewitt, J. K. y Friedman, N. P. (2017). Executive functions and substance use: Relations in late adolescence and early adulthood. Journal of Abnormal Psychology, 126, 257-270. doi:10.1037/abn0000250.

Hall, F. S., Der-Avakian, A., Gould, T. J., Markou, A., Shoaib, M. y Young, J. W. (2015). Negative affective states and cognitive impairments in nicotine dependence. Neuroscience E Biobehavioral Reviews, 58, 168-185. doi:10.1016/j. neubiorev.2015.06.004.

Harakeh, Z., de Sonneville, L., van den Eijnden, R. J. J. M., Huizink, A. C., Reijneveld, S. A., Ormel, J.,... Vollebergh, W. A. M. (2012). The association between neurocognitive functioning and smoking in adolescence: The TRAILS study. Neuropsychology, 26, 541-550. doi:10.1037/ a0029217.

Harvanko, A. M., Strickland, J. C., Slone, S. A., Shelton, B. J. y Reynolds, B. A. (2019). Dimensions of impulsive behavior: Predicting contingency management treatment outcomes for adolescent smokers. Addictive Behaviors, 90, 334-340. doi:10.1016/j.addbeh.2018.11.031.

Heishman, S. J., Kleykamp, B. A. y Singleton, E. G. (2010). Meta-analysis of the acute effects of nicotine and smoking on human performance. Psychopharmacology, 210, 453-469. doi:10.1007/s00213-010-1848-1.

Helmstaedter, C., Lendt, M. y Lux, S. (2001). Verbaler Lernund Merkfähigkeitstest: VLMT. Manual. Beltz-Test.

Hester, R. y Garavan, H. (2009). Neural mechanisms underlying drug-related cue distraction in active cocaine users. Pharmacology Biochemistry and Behavior, 93, 270277. doi:10.1016/j.pbb.2008.12.009.

Hu, P., Huang, L., Zhou, S., Shi, Q., Xiao, D. y Wang, C. (2018). Smoking status and cognitive performance among vocational school students in Beijing, China. Respiratory Medicine, 135, 8-11. doi:10.1016/j. rmed.2017.12.008.

Kirby, K. N., Petry, N. M. y Bickel, W. K. (1999). Heroin addicts have higher discount rates for delayed rewards than non-drug-using controls. Journal of Experimental Psychology: General, 128, 78-87. doi:10.1037/0096-3445.128.1.78.

Krishnan-Sarin, S., Reynolds, B., Duhig, A. M., Smith, A., Liss, T., McFetridge, A.,... Potenza, M. N. (2007). Behavioral impulsivity predicts treatment outcome in a smoking cessation program for adolescent smokers. Drug and Alcohol Dependence, 88, 79-82. doi:10.1016/j.drugalcdep.2006.09.006. 
Kübler, A., Murphy, K. y Garavan, H. (2005). Cocaine dependence and attention switching within and between verbal and visuospatial working memory. European Journal of Neuroscience, 21, 1984-1992. doi:10.1111/j.14609568.2005.04027.x.

Li, Y., Yuan, K., Cai, C., Feng, D., Yin, J., Bi, Y.,... Tian, J. (2015). Reduced frontal cortical thickness and increased caudate volume within fronto-striatal circuits in young adult smokers. Drug and Alcohol Dependence, 151, 211-219. doi:10.1016/j.drugalcdep.2015.03.023.

López-Torrecillas, F. (1996). Estrés, afrontamiento, variables de personalidad y consumo de drogas. Granada: Universidad de Granada. Recuperado de http://hdl.handle. net/10481/14874.

López-Torrecillas, F., Perales, J. C., Nieto-Ruiz, A. y Verdejo-García, A. (2014). Temperament and impulsivity predictors of smoking cessation outcomes. PLoS ONE, 9, e112440. doi:10.1371/journal.pone.0112440.

Luijten, M., Kleinjan, M. y Franken, I. H. A. (2016). Event-related potentials reflecting smoking cue reactivity and cognitive control as predictors of smoking relapse and resumption. Psychopharmacology, 233, 2857-2868. doi:10.1007/s00213-016-4332-8

Lydon, D. M., Wilson, S. J., Child, A. y Geier, C. F. (2014). Adolescent brain maturation and smoking: What we know and where we're headed. Neuroscience and Biobehavioral Reviews, 45, 323-342. doi:10.1016/j.neubiorev.2014.07.003.

Lyvers, M., Carlopio, C., Bothma, V. y Edwards, M. S. (2014). Mood, mood regulation, and frontal systems functioning in current smokers, long-term abstinent ex-smokers, and never-smokers. Journal of Psychoactive Drugs, 46, 133-139. doi:10.1080/02791072.2013.876522.

Myerson, J., Green, L. y Warusawitharana, M. (2001). Area under the curve as a measure of discounting. Journal of the Experimental Analysis of Behavior, 76, 235-243. doi:10.1901/jeab.2001.76-235.

Newhouse, P., Kellar, K., Aisen, P., White, H., Wesnes, K., Coderre, E.,... Levin, E. (2012). Nicotine treatment of mild cognitive impairment. Neurology, 78, 91-101. doi:10.1212/WNL.0b013e31823efcbb.

O’Dell, L. E. y Torres, O. V. (2014). A mechanistic hypothesis of the factors that enhance vulnerability to nicotine use in females. Neuropharmacology, 76, 566-580. doi:10.1016/j.neuropharm.2013.04.055.

Paulus, M. P. y Stewart, J. L. (2014). Interoception and drug addiction. Neuropharmacology, 76(PART B), 342350. doi:10.1016/j.neuropharm.2013.07.002.

Reitan, R. M. (1958). Validity of the Trail Making Test as an indicator of organic brain damage. Perceptual and Motor Skills, 8, 271-276. doi:10.2466/pms.1958.8.3.271.

Reynolds, B. y Fields, S. (2012). Delay discounting by adolescents experimenting with cigarette smoking. Addiction, 107, 417-424. doi:10.1111/j.1360-0443.2011.03644.x.
Reynolds, B., Richards, J. B., Horn, K. y Karraker, K. (2004). Delay discounting and probability discounting as related to cigarette smoking status in adults. Behavioural Processes, 65, 35-42. doi:10.1016/S0376-6357(03)00109-8.

Sheffer, C. E., Christensen, D. R., Landes, R., Carter, L. P., Jackson, L. y Bickel, W. K. (2014). Delay discounting rates: A strong prognostic indicator of smoking relapse. Addictive Behaviors, 39, 1682-1689. doi:10.1016/j.addbeh.2014.04.019.

Stroop, J. R. (1935). Studies of interference in serial verbal reactions. Journal of Experimental Psychology, 18, 643-662. doi:10.1037/h0054651.

Sutherland, M. T., Ray, K. L., Riedel, M. C., Yanes, J. A., Stein, E. A. y Laird, A. R. (2015). Neurobiological impact of nicotinic acetylcholine receptor agonists: An activation likelihood estimation meta-analysis of pharmacologic neuroimaging studies. Biological Psychiatry, 78, 711-720. doi:10.1016/j.biopsych.2014.12.021.

Sutherland, M. T., Riedel, M. C., Flannery, J. S., Yanes, J. A., Fox, P. T., Stein, E. A. y Laird, A. R. (2016). Chronic cigarette smoking is linked with structural alterations in brain regions showing acute nicotinic drug-induced functional modulations. Behavioral and Brain Functions, 12, 16. doi:10.1186/s12993-016-0100-5.

Sutherland, M. T. y Stein, E. A. (2018). Functional neurocircuits and neuroimaging biomarkers of tobacco use disorder. Trends in Molecular Medicine, 24, 129-143. doi:10.1016/j.molmed.2017.12.002.

Trenerry, M.R., Crosson, B., Deboe, J. y Leber, W. R. (1990). Visual Search and Attention Test. Psychological Assessment Resources, Inc. Odessa, Florida.

Valentine, G. y Sofuoglu, M. (2018). Cognitive effects of nicotine: Recent progress. Current Neuropharmacology, 16, 403-414. doi:10.2174/1570159X15666171103152136.

Verdejo-García, A., Alcázar-Córcoles, M. A. y Albein-Urios, N. (2019). Neuropsychological interventions for decision-making in addiction: A systematic review. Neuropsychology Review, 29, 79-92. doi:10.1007/s11065-018-9384-6.

Villalbi, J. R., Suelves, J. M., Martínez, C., Valverde, A., Cabezas, C. y Fernández, E. (2019). El control del tabaquismo en España: Situación actual y prioridades. Revista Española de Salud Pública, 93, e1-e6.

Wagner, M., Schulze-Rauschenbach, S., Petrovsky, N., Brinkmeyer, J., von der Goltz, C., Gründer, G.,... Winterer, G. (2013). Neurocognitive impairments in non-deprived smokers-results from a population-based multi-center study on smoking-related behavior. Addiction Biology, 18, 752-761. doi:10.1111/j.1369-1600.2011.00429.x.

Wechsler, D. (1999). WAIS-III. Escala de inteligencia de Wechsler para adultos -III. Madrid.

World Health Organization. (2019). WHO Report on Global Tobacco Epidemic. Recuperado de https://doi.org/ https://www.who.int/news-room/fact-sheets/detail/ tobacco. 
Zarrindast, M.-R. y Khakpai, F. (2019). The modulatory role of nicotine on cognitive and non-cognitive functions. Brain Research, 1710, 92-101. doi:10.1016/j.brainres.2018.12.002.

Zlomuzica, A., Machulska, A., Roberts, S., Von Glischinski, M., Rinck, M., Lester, K. J.,... Margraf, J. (2018). The dopamine D2 receptor mediates approach-avoidance tendencies in smokers. European Archives of Psychiatry and Clinical Neuroscience, 268, 261-268. doi:10.1007/s00406017-0793-y. 
